\title{
Solving Linear and Non-Linear Stiff System of Ordinary Differential Equations by Multistage Adomian Decomposition Method
}

\author{
M. S. H. Chowdhury, I. Hashim, Md. Alal Hosen
}

\begin{abstract}
In this paper, linear and non-linear stiff systems of ordinary differential equations are solved by the classical Adomian decomposition method (ADM) and the multistage Adomian decomposition method (MADM). The MADM is a technique adapted from the standard Adomian decomposition method (ADM) where standard ADM is converted into a hybrid numeric-analytic method called the multistage ADM (MADM). The MADM is tested for several examples. Comparisons with an explicit Runge-Kutta-type method (RK) and the classical ADM demonstrate the limitations of ADM and promising capability of the MADM for solving stiff initial value problems (IVPs).
\end{abstract}

Keywords-- Stiff system of ODEs, Runge-Kutta-type method, Adomian decomposition method, Multistage ADM.

\section{Introduction}

The mathematical equations modelling many real-world physical phenomena are often stiff equations, i.e. equations with a wide range of temporal scales. The numerical methods for solving stiff equations must have good accuracy and wide region of stability. Hojjati et al. [1] developed a multistep method for solving stiff systems of initial value problems (IVPs). Knowing that the classical explicit fourth-order Runge-Kutta method is insufficient for the solution of stiff IVPs, Ahmad et al. [2] presented an explicit Taylor-like method for solving stiff IVPs. In Ahmad and Yaacob [3], an explicit Runge-Kutta-like method is developed and shown to be efficient for the solution of stiff ODEs. Very recently, Nie et al. [4] presented a class of efficient semi-implicit schemes for stiff reaction-diffusion equations.

\section{S. H. Chowdhury}

Department of Science in Engineering, Faculty of Engineering, International Islamic University Malaysia, Jalan Gombak, 53100 Kuala Lumpur, Malaysia. Corresponding author: M. S. H. Chowdhury

\section{Hashim}

School of Mathematical Science, Faculty of Science and Technology, Universiti Kebangsaan Malaysia.

\section{Md. Alal Hosen}

Present: Department of Manufacturing and Materials Engineering, Faculty of Engineering, International Islamic University Malaysia, Jalan Gombak, 53100 Kuala Lumpur, Malaysia.

Past: Department of Mathematics, Rajshahi University of Engineering and Technology, Rajshahi-6204, Bangladesh.

A variable-step size algorithm for stiff systems has been proposed recently by Jannelli and Fazio [5]. In [6], classes of methods having properties very close to those of traditional Runge-Kutta methods were developed. Butcher and Hojjati [7] devised a class of second derivative methods possessing Runge-Kutta stability property. Hojjati et al. [8] presented a new class of second derivative multistep methods with improved stability region.

All of the methods mentioned above need some sort of discretizations. One of the papers proposing an approximate analytic method is due to Guzel and Bayram [9] who presented a power series method for stiff systems. Adomian et al. [10] first demonstrated how a power series method, called the Adomian decomposition method (ADM) [11], can be used to derive an exact solution to a specific linear stiff system of IVPs. The ADM yields, without linearization, perturbation, transformation or discretization, an analytical solution in terms of a rapidly convergent infinite power series with easily computable terms, see for example, [11]. Recently, Mahmood et al. [12] solved both linear and non-linear stiff systems of IVPs using the ADM. The ADM has been applied to a wide range of problems, [13, 14, 15, 16, 17, 18]. In [19, 20, 21, 22, $23,24]$, the ADM was treated as an algorithm for approximating the solutions in a sequence of time intervals (i.e. time step). We call this approach as multi-stage ADM (MADM). In this work, we shall apply the MADM to the solutions of stiff IVPs. Comparisons will be made against the classical ADM, an explicit Runge-Kutta method and available exact solutions.

\section{Solution Methods}

In this section we describe the ADM and give the algorithm of the Runge-Kutta-like method of Ahmad and Yaacob [3] for solving the following initial value problem:

$$
y^{\prime}=f(t, y) \text { with } y(0)=y_{0},
$$

where $f(t, y)$ may be a linear or non-linear function.

\section{A. Decomposition method}

Consider Eq. (1) written in the form

$$
L_{y}+R_{y}+N_{y}=g(t),
$$

where $L=d / d t, R$ is the remainder of the linear operator and $N_{y}$ represents the non-linear term. Hence, we obtain

$$
y=y(0)+L^{-1}(g(t))-L^{-1}\left(R_{y}\right)-L^{-1}\left(N_{y}\right),
$$

where $L^{-1}(\cdot)=\int_{0}^{t}(\cdot) d s$. The ADM, [13], takes the solution $y$ and the non-linear function $N_{y}$ as infinite series, respectively, 


$$
y=\sum_{k=0}^{\infty} y_{k}, \quad N_{y}=\sum_{k=0}^{\infty} A_{k} .
$$

The $y_{k}{ }^{\prime} s$ are determined from the recursive algorithm, [13], based on (3),

$$
\begin{aligned}
& y_{0}=y(0)+L^{-1}(g(t)), \\
& y_{k+1}=-L^{-1}\left(R_{y_{k}}\right)-L^{-1}\left(A_{y_{k}}\right), \quad k \geq 0,
\end{aligned}
$$

where the so-called Adomian polynomials $A_{k}$ are given by, [13],

$$
A_{K}=\frac{1}{k !}\left[\frac{d^{k}}{d \lambda^{k}} N\left(\sum_{j=0}^{\infty} \lambda^{j} y_{j}\right)\right]_{\lambda=0}, \quad k=0,1,2, \ldots \ldots
$$

Convergence aspects of the ADM have been investigated in [25]. For later numerical computation, let the expression

$$
\phi_{n}(t)=\sum_{k=0}^{n-1} y_{k}
$$

denote the $n$-term approximation to $y(t)$.

As first hinted in [26] and applied in [19, 20, 21, 22, 23], we treat the $\mathrm{ADM}$ as an algo-rithm for approximating the dynamical response in a sequence of time intervals (i.e. time step) $\left[0, t_{1}\right),\left[t_{1}, t_{2}\right), \ldots,\left[t_{m-1}, T\right)$ such that the initial condition in $\left[t^{*}, t_{m+1}\right]$ is taken to be the condition at $t^{*}$.

\section{в. Runge-Kutta-like method}

Ahmad and Yaacob [3] developed composite arithmeticharmonic, explicit Runge-Kutta-like methods for solving problem (1). The iterative formula they proposed is given as follows,

$$
\begin{aligned}
& y_{n+1}=y_{n}+\frac{h}{2}\left(\frac{2 k_{1} k_{2}}{k_{1}+k_{2}}+\frac{2 k_{2} k_{3}}{k_{2}+k_{3}}\right), \\
& k_{1}=f\left(t_{n}, y_{n}\right), \\
& k_{2}=f\left(t_{n}+\frac{3}{5} h, y_{n}+\frac{3}{5} h k_{1}\right),
\end{aligned}
$$

$$
k_{3}=f\left(t_{n}+\frac{4}{5} h, y_{n}+\frac{4}{5} h\left[\frac{k_{1}+k_{2}}{2}\right]\right),
$$

where $h$ is the time stepsize. For further details the reader is referred to [3].

\section{Test Problems}

In this section, we shall demonstrate how well the MADM compares with the RungeKutta-like method of [3] for the solutions of both linear and non-linear system of ordinary differential equations (ODES). The Adomian iterative algorithm is coded in the computer algebra package Maple. The Maple environment variable Digits controlling the number of significant digits is set to 16 in all the calculations done in this paper.

\section{A. Problem 1}

Consider linear stiff initial value problem [28]:

$$
\begin{aligned}
& \frac{d y_{1}}{d t}=-0.1 y_{1}-49.9 y_{2}, \\
& \frac{d y_{2}}{d t}=-50 y_{2}, \\
& \frac{d y_{3}}{d t}=70 y_{2}-120 y_{3},
\end{aligned}
$$

subject to the initial conditions

$$
y_{1}(0)=2, \quad y_{2}(0)=1, \quad y_{3}(0)=2 .
$$

The exact solutions of the system (13)-(15) are given by

$$
\begin{aligned}
& y_{1}(t)=e^{-50 t}+e^{-0.1 t}, \\
& y_{2}(t)=e^{-50 t}, \\
& y_{3}(t)=e^{-50 t}+e^{120} .
\end{aligned}
$$

The iterative formula based on (5) and (6) for the system (13)-(15) are given by

$$
\begin{aligned}
& y_{1,0}=2, \quad y_{2,0}=1, \quad y_{3,0}=2, \\
& y_{1, k+1}=-0.1 L^{-1}\left(y_{1, k}\right)-49.9 L^{-1}\left(y_{2, k}\right), \quad k \geq 0, \\
& y_{2, k+1}=-50 L^{-1}\left(y_{2, k}\right), \quad k \geq 0, \\
& y_{3, k+1}=70 L^{-1}\left(y_{2, k}\right)-120 L^{-1}\left(y_{3, k}\right), \quad k \geq 0 .
\end{aligned}
$$

From Fig 1, it is observed that the 4-term MADM solutions agree very well with the exact solutions.

\section{A. Problem 2}

Now consider nonlinear stiff initial value problem [29]

$$
\begin{aligned}
& \frac{d y_{1}}{d t}=-12 y_{1}+10 y_{2}^{2}, \\
& \frac{d y_{2}}{d t}=y_{1}-y_{2}-y_{1} y_{2},
\end{aligned}
$$

subject to the initial conditions

$$
y_{1}(0)=0, \quad y_{2}(0)=0 \text {. }
$$

The exact solutions of the system (24)-(25) are given by

$$
\begin{aligned}
& y_{1}(t)=e^{-2 t}, \\
& y_{2}(t)=e^{-t} .
\end{aligned}
$$

The iterative formula based on (5) and (6) for the system (24)-(25) are given by

$$
\begin{gathered}
y_{1,0}=0, \quad y_{2,0}=0, \\
y_{1, k+1}=-12 L^{-1}\left(y_{1, k}\right)+10 L^{-1}\left(A_{k}\right), \quad k \geq 0, \\
y_{2, k+1}=L^{-1}\left(y_{1, k}\right)-L^{-1}\left(B_{k}\right), \quad k \geq 0,
\end{gathered}
$$

where some of the Adomian polynomials for $A_{k}$ and $B_{k}$ obtained from (7) are given bellow:

$$
A_{0}=y_{1,0}^{2}, \quad A_{1}=2 y_{1,0} y_{2,0}, \quad A_{2}=2 y_{1,0} y_{1,2}+y_{1,1}^{2},
$$

etc.

$B_{0}=y_{1,0} y_{2,0}, B_{1}=y_{1,0} y_{2,1}+y_{1,1} y_{2,0}, B_{2}=y_{1,0} y_{2,2}+y_{1,1} y_{2,1}+y_{1,2} y_{2,0}$ ete.

In Figure 2 we compare the 4-term decomposition solutions with the exact solutions and that obtained by the Runge-Kutta method. The MADM solutions at $h=10^{-3}$ are of comparable 
accuracy with the exact solutions and that of the Runge-Kutta method at the same step size.
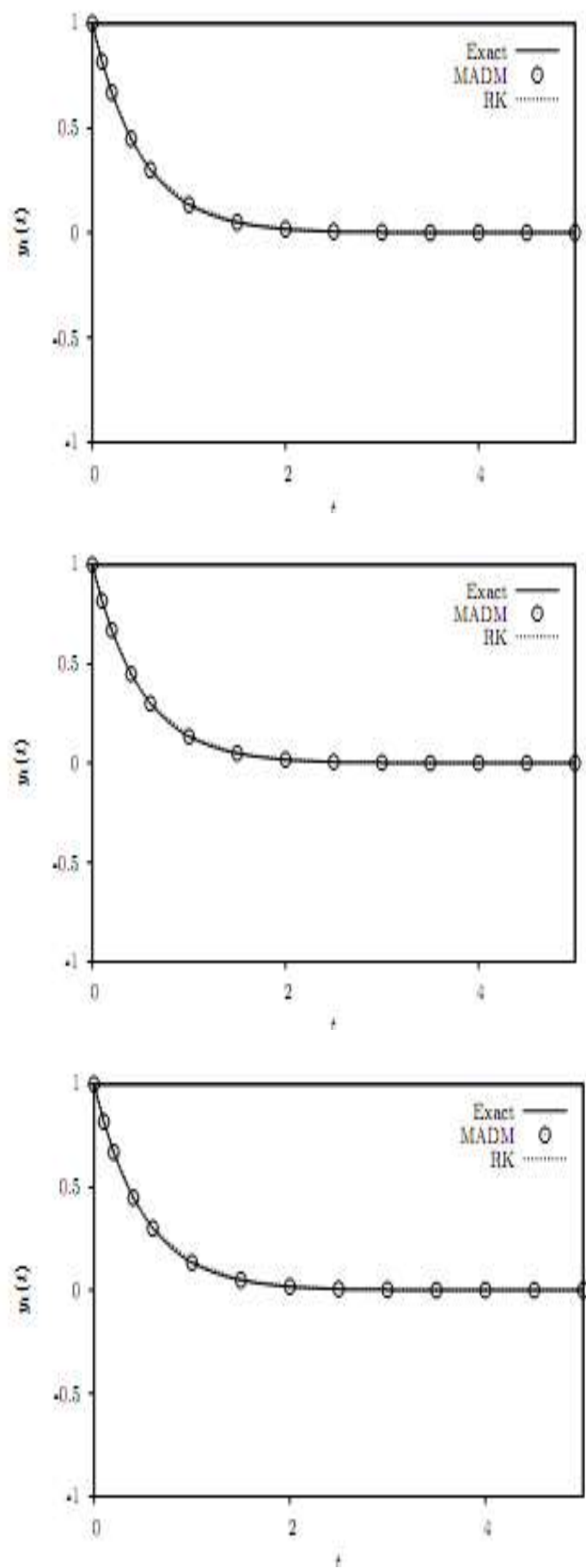

Figure 1: The MADM solutions using 4 terms at time step $h=10^{-3}$ as compared with the exact solutions for Problem 1.
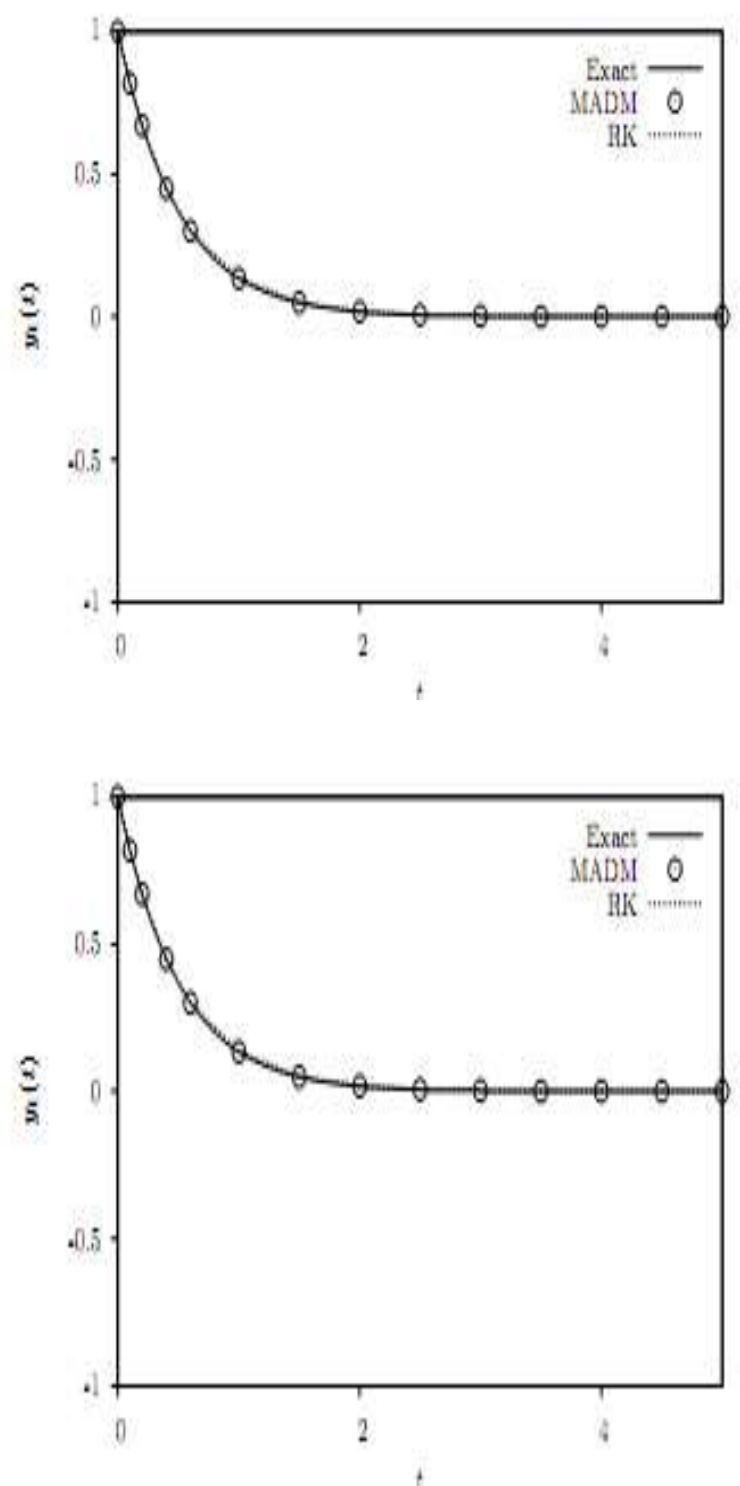

Figure 2: The MADM solutions using 4 terms as compared with the exact solutions and that obtained by the Runge-Kutta method for Problem 2.

\section{Conclusion}

In this paper, we presented the multi-stage Adomian decomposition method (MADM) for solving both linear and non-linear stiff system of ODEs. Direct applications of the classical ADM can fail for stiff problems. The MADM is shown here to be a promising alternative method for stiff equations. In addition to the choice of time stepsize, the MADM has the number of terms of the series solution as an extra parameter for controlling the accuracy of solutions. 


\section{Acknowledgement}

The authors would like to acknowledge the financial supports received from the International Islamic University Malaysia, Ministry of higher education Malaysia through the research grant FRGS14-143-0384.

\section{References}

[1] G. Hojjati, M. Y. Rahimi-Ardabili and S.M. Hosseini, "A-EBDF: an adaptive method for numerical solution of stiff systems of ODEs," Mathematics and Computers in Simulation, vol. 66(1), pp.33-41, 2004.

[2] R. R. Ahmad, N. Yaacob and A. H. Mohd-Murid, "Explicit methods in solving stiff ordinary differential equations," International Journal of Computer Mathematics, vol. 81(11), pp. 1407-1415, 2004.

[3] R. R. Ahmad and N. Yaacob, "Third-order composite Runge-Kutta method for stiff problems," International Journal of Computer Mathematics, vol. 82(10), pp. 12211226, 2005.

[4] Q. Nie, Y. T. Zhang and R. Zhao, "Efficient semi-implicit schemes for stiff systems," Journal of Computational Physics, vol. 214(9), pp. 521537, 2006.

[5] A. Jannelli and R. Fazio, "Adaptive stiff solvers at low accuracy and complexity," Journal of Computational and Applied Mathematics, vol. 191(2), pp. 246-258, 2006.

[6] J. C. Butcher and N. Rattenbury, "ARK methods for stiff problems," Applied Numerical Mathematics, vol. 53(2-4), pp. 165-181, 2005.

[7] J. C. Butcher and G. Hojjati, "Second derivative methods with RK stability," Numerical Algorithms, vol. 40(4), pp.415-429, 2005.

[8] G. Hojjati, M. Y. Rahimi-Ardabili and S. M. Hosseini, "New second derivative multistep methods for stiff systems," Applied Mathematical Modelling, vol. 30(5), pp. 466-476, 2006.

[9] N. Guzel and M. Bayram, "On the numerical solution of stiff systems," Applied Mathematics and Computation , vol. 170(1), pp. 230-236, 2005.

[10] G. Adomian, R. Rach and M. Elrod, "The decomposition method applied to stiff systems," Mathematics and Computers in Simulation, vol. 30(3), pp. 271-276, 1988.

[11] G. Adomian, "A review of the decomposition method in applied mathematics," Journal of Mathematical Analysis and Applications, vol. 135(2), pp. 501-544, 1988 .

[12] A. S. Mahmood, L. Casasus and W. Al-Hayani, "The decomposition method for stiff systems of ordinary differential equations," Applied Mathematics and Computation, vol. 167(2), pp. 964-975, 2005.

[13] G. Adomian, Solving frontier problems of physics: the decomposition method. Kluwer Academic, Boston, 1994.

[14] I. Hashim, "Adomian decomposition method for solving BVPs for fourth-order integro-differential equations," Journal of Computational and Applied Mathematics, vol. 193(2), pp. 658-664, 2006.

[15] I. Hashim, M. S. M. Noorani, and M. R. Said Al-Hadidi, "Solving the generalized Burgers-Huxley equation using the Adomian decomposition method," Mathematical and Computer Modelling, vol. 43(11-12), pp. 1404-1411, 2006

[16] D. Kaya and S. M. El-Sayed, "Numerical soliton-like solutions of the potential Kadomtsev-Petviashvili equation by the decomposition method," Physics Letters A, vol. 320(2-3), pp. 192-199, 2003.

[17] L. Wang, "A new algorithm for solving classical Blasius equation," Applied Mathematics and Computation, vol. 157(1), pp. 1-9, 2004.

[18] A. M. Wazwaz, "Exact solutions with solitons and periodic structures for the Zakharov-Kuznetsov (ZK) equation and its modified form," Communications in Nonlinear Science and Numerical Simulation, vol. 10(6), pp. 597-606, 2005.

[19] S. Guellal, P. Grimalt and Y. Cherruault, "Numerical study of Lorenz's equation by the Adomian method," Computers and Mathematics with Applications, vol. 33(3), pp. 25-29, 1997.

[20] S. Olek, "An accurate solution to the multispecies Lotka-Volterra equations," SIAM Review, vol. 36(3), pp. 480-488, 1994.

[21] N. Shawagfeh and D. Kaya, "Comparing numerical methods for the solutions of systems of ordinary differential equations," Applied Mathematics Letters, vol. 17(3), pp. 323-328, 2004.

[22] P. Vadasz and S. Olek, "Convergence and accuracy of Adomian's decomposition method for the solution of Lorenz equation,"
International Journal of Heat and Mass Transfer, vol. 43(10), pp. 1715-1734, 2000.

[23] I. Hashim, M. S. M. Noorani, R. Ahmad, S. A. Bakar, E. S. Ismail and A. M. Zakaria, "Accuracy of the Adomian decomposition method applied to the Lorenz system," Chaos, Solitons \& Fractals, vol. 28(5), pp. 1149-1158, 2006.

[24] M. S. H. Chowdhury, I. Hashim and S. Mawa, "Solution of preypredator problem by numericanalytic technique," Commun. Nonlin. Sci. Numer. Simul., vol. 14, pp. 1008-1012, 2009.

[25] K. Abbaoui and Y. Cherruault, "New ideas for proving convergence of decomposition methods," Computers and Mathematics with Applications, vol. 29(7), pp. 103-108, 1995.

[26] G. Adomian, Nonlinear stochastics systems theory and application to physics. Reidel, Dordrecht, 1988.

[27] M. E. Fowler and R. M. Warten, "A numerical integration technique for ordinary differential equations with widely separated eigenvalues," IBM Jour., vol. 11, pp. 537543, 1967.

[28] J. R. Cash, "A fortran subroutine for solution of first order systems of ordinary differential equations," ACM. Trans., vol. 18 (2), pp. 156158, 1992.

[29] P. Kaps, Rosenbrock-type methods, in: G. Dahalquist, R. Jeltsch (Eds), Numerical methods for stiff initial value problems. Bericht Nr.9, Inst. fur geometrie und Praktische Mathematik der RWTH Aachen, 1981. 